\title{
Autonomous Monitoring of River Level with Real Time Event Prediction
}

\author{
Zamshed I. Chowdhury ${ }^{1}$, Md. Istiaque Rahaman ${ }^{2} \&$ Shahriar I. Chowdhury $^{3}$ \\ ${ }^{1}$ Institute of Information Technology, Jahangirnagar University, Savar, Dhaka, Bangladesh \\ ${ }^{2}$ Department of Electrical \& Electronic Engineering, Ahsanullah University of Science \& Technology, Dhaka, \\ Bangladesh \\ ${ }^{3}$ Desme Bangladesh, Dhaka, Bangladesh \\ Correspondence: Zamshed I. Chowdhury, Institute of Information Technology, Jahangirnagar University, Savar, \\ Dhaka-1342, Bangladesh. Tel: 880-277-910-4551. E-mail: zic@juniv.edu
}

\author{
Received: April 9, $2014 \quad$ Accepted: April 22, $2014 \quad$ Online Published: July 29, 2014 \\ doi:10.5539/cis.v7n3p67 URL: http://dx.doi.org/10.5539/cis.v7n3p67
}

\begin{abstract}
Observation of water level at various river sites could provide valuable insight about probable disaster in advance to initiate disaster management protocol as early as possible. We have developed an autonomous remote river water level monitoring network with event prediction algorithm at the server while maintaining a substantially low manufacturing cost. The WSN is comprised of several chosen sites based on their statistics with intelligent sensors for water level measurement. The sensors are autonomous in nature to account for any disturbance in node environment and also within the network. The real time data are transmitted to a remote server through GPRS for further processing. Server extracts information and simulates various real time parameters such as water level rise rate, time remaining to exceed the critical level for a particular site etc. A prediction algorithm running on the server side predicts the measured level values for each node over a period of time. A prototype system is implemented with six nodes at different points and has yielded satisfactory results.
\end{abstract}

Keywords: data acquisition module, flood, GSM network, PIC18f4550, prediction analysis, real time, river level, WSN

\section{Introduction}

In coastal countries where rivers are flowing everywhere through lands, flood is considered to be one of the most common natural disasters. About 100,000 persons are killed and over 1.4 billion people are affected during the last decade of the 20th century as described by S. N. Jonkman (Jonkman, 2005). Continuous monitoring of river water level at alarming points could provide with valuable information that can be utilized to warn authorities and inhabitants well ahead in time. A review on conventional river water level measuring showed that river level monitoring by constructing piezometer on the location under investigation is somewhat costly and requires human presence for data collection (Winter, LaBaugh, \& Rosenberry, 1988). Therefore, information cannot be gathered ahead in time. The modern sensor network for this purpose has pressure sensors installed at different points to acquire data with high precision. But the use of this kind of networks is limited by the cost involved with installation and maintenance. For developing and under developed countries where such costly method is not a viable option, the threat of mass destruction by sudden flood is still at large. The only solution is to find a low cost system that could provide a near precision measurement and timely warning so that if water level is about to exceed the critical level, concerning authorities might be able to be prepared to minimize the figure of flood caused deaths and immense harms. This paper illustrates such an autonomous wireless sensor network (WSN) for twenty four hour river water level surveillance and alarming tasks.

This article is a revised and expanded version of a paper entitled Design and Deployment of a Robust Remote River Level Sensor Network, presented at IEEE Sensors Applications Symposium, TX, USA, February 22-24, 2011(Chowdhury et al., 2011). The sensor network architecture is illustrated using a block diagram shown in figure 1. At the heart of this network, a server collects data periodically sent from installed nodes (Node 1, Node $2 \ldots$ Nodes $\mathrm{N}$ ) placed at different sites along the rivers. The nodes are responsible for raw data collection, storage and transmission to the server after initial processing. Existing GSM (Global System for Mobiles) network is 
used to connect the nodes to a server. Connecting wireless sensors together through the use of GSM network is a widely used technique to build wireless sensor networks. Some notable works have been done on sensor network for distance measurement. One such work is the measurement of distance using time of flight and phase shift method (Gueuning et al., 1996). Sensor networks are designed as distributed network (Cardell-Oliver et al., 2005) as well as centralized network using WLAN (Postolache et al., 2005). The nodes are data acquisition systems that are installed near bank of the river where depth is moderate. These embedded systems are designed to operate without direct human supervision. The data are uploaded by the nodes through GPRS (General Packet Radio Service) to a remote server. The server which is designated as Central Monitoring Unit (CMU) is basically a database software which receives data, extract information and further processes the information based on natural criteria and estimates critical parameters. These newly obtained parameters are refreshed periodically and updated as soon as new raw data are received from the sensing nodes. Based on the prior information, CMU predicts values of critical parameters for each node. If the levels approach critical values at a constant rate or rise suddenly, then sensors would sample the water level information with a shorter acquisition period to be at a high priority state or to detect any sudden change.

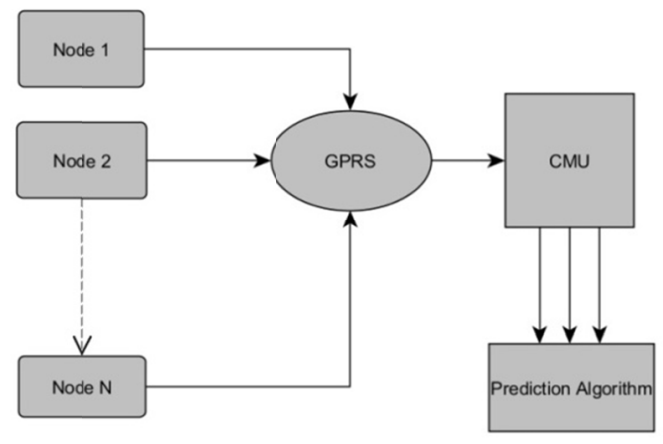

Figure 1. Network architecture block diagram

This article is organized as follows: the whole system architecture from both hardware and software perspectives are presented in section 2. In the next section, the remote server design process and the associated algorithms are presented. The result and analysis section discusses the results from the implemented prototype and the concluding section discusses the future improvements.

\section{System Architecture}

\subsection{Basis of Distance Measurement and Vertical Distance}

The concept behind the vertical distance measurement is very similar to the one using ultrasonic (US) signal for horizontal distance measurement. This is a well-known concept which is first brought into attention by M. Toda and K. T. Park (1998). The data acquisition system used here adopts the similar technique but for measuring vertical distance. The measurement module contains an ultrasonic transmitter-receiver pair, a processor for calculating the node entities (in this case, a microcontroller), real-time clock and non-volatile data memory to compute and store yielded water level data. The module block diagram is shown in figure 2 . The US transducer converts electrical signal to US signal and vice versa. The transmitter is a piezo-electric crystal which receives a signal having a frequency equal to the characteristic frequency of the crystal and converts it to an equivalent US signal of the same frequency and transmits it. The US signal reflects back from any obstacle that the signal cannot pass through and is received by another crystal termed as receiver. This crystal converts the signal back to an equivalent electronic signal of the source frequency. The processor governs the transmission and reception of US signals and uses a timer and capture module to get the time elapsed between the transmission and the reception of echo. The sound wave travels through air at a velocity of $340 \mathrm{~ms}^{-1}$. If the timer count is $t$ seconds after the transmission has occurred and till the reception, the horizontal distance of the obstacle from the module should be, $l=(340 \times t) / 2$ meters. The factor of 2 comes from the fact that the transmitted signal must traverse twice the length to get to the receiver from the transmitter. Each measured level is marked using real time stamp. The resulting data is stored into the EEPROM by the processor for later processing which includes sending data periodically to a remote server and for data recovery, in case of a delivery failure.

\subsection{The Mounting Structure}

The data acquisition system and the GSM network interfacing circuit is integrated into a single module and is 
mounted on top of a tube like structure made of PVC. The module is oriented vertically from the surface with the transmitter and receiver both facing downward (towards the ground surface). The tube has two openings: one near the bottom end and the other near the top end. The purpose of two separate windows is that water does not experience the air pressure building inside the tube as water enters and compresses the air column inside. As a result, the water level remains at the same level as outside of the tube. Each mounting structure and associated module is placed at different chosen sites. When the tube is placed at a node from where data is to be collected, the water enters the tube and floats a hollow box made of plastic having a thickness of $5 \mathrm{~cm}$ which was already inside the tube. The thickness is chosen to be enough to provide an opaque medium for the ultrasonic signal which could reflect back the incident US signal. A radius of $10 \mathrm{~cm}$ makes the box move up and down the length of the tube almost without any friction as the tube has a diameter of $12 \mathrm{~cm}$. This box acts as the obstacle for the data acquisition system. When the bottom end of the tube is sunk for the first time, water flows into the tube through the window near the river bottom. The water continues to rise in the tube until the water levels inside and outside the tube become equal. The whole arrangement is shown in figure 3 as suggested in the previous work (Chowdhury et al., 2011).

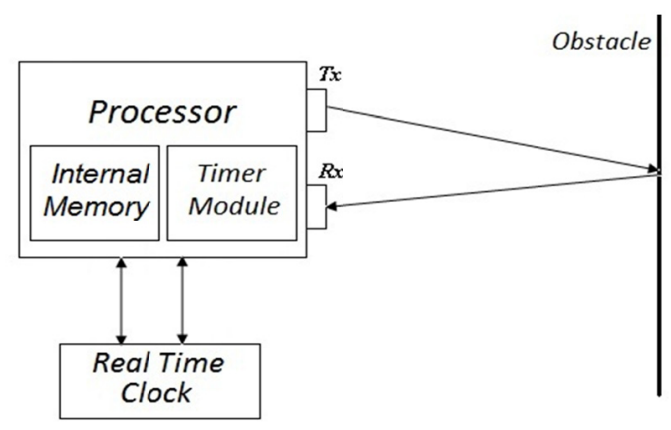

Figure 2. Data Acquisition System (DAS) block diagram

Once the vertical height is being measured, the water column height is calculated by excluding the bias values which are in fact the physical parameters associated with the tube. It is evident that the data acquisition system measures the distance from the module to the floating box on the water surface which is denoted by $x$ meter. The thickness of the box is $0.05 \mathrm{~m}$ and the height of the tube from the module to the bottom surface is $L$ meter. If the height of the slab above the water is $d$ meter, the effective water column height is $h=(L-x-d)$ meter. The value of $h$ is calculated at node site and transferred to the CMU for further processing. The portion of the box that stays above the water surface varies from site to site due to the variation in density of the water at different sites. The height should be more than what it is at a site which is nearer to sea water as sea water is more content with different types of salt. This particular dimension is determined manually at each site prior to the installation and included in the firmware of the each of the node processors to calculate effective height of water column.

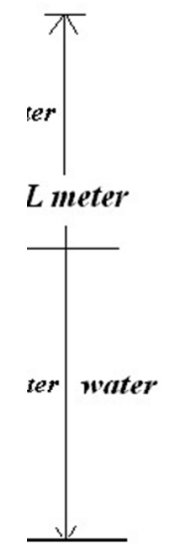

Figure 3. Mounting structure 


\subsection{Node Hardware Architecture}

The hardware is designed following a modular approach. Each node module comprised of four sub-modules and finally assembled into a single module. Each of the sub-modules is designed for specific functionality as mentioned below:

1) Data Acquisition Module (DAM): The distance measuring section and the associated circuitry form DAM. DAM incorporates several hardware blocks inside a DAM as illustrated in figure 4:

i) US signal generator and transmitter block

ii) US signal reception and processing block and

iii) Data processing and storage block

US signal generator and transmitter block consists of a US transmitter $\left(\mathrm{T}_{\mathrm{X}}\right)$, a multi-vibrator and additional circuit elements. NE555N provides the oscillation needed for the transmitter. The inverter is used for the drive of $\mathrm{T}_{\mathrm{X}}$. Two hex inverters are connected in parallel to increase the strength of the transmitted pulse. By cutting dc current with capacitor, about twice the voltage from the inverter output is applied to the transmitter. The frequency of the signal and the whole generation process is controlled by a microcontroller. US signal reception and processing block includes a US receiver $\left(\mathrm{R}_{\mathrm{X}}\right)$, an amplifier and a threshold detector. The ultrasonic signal which was received by $R_{X}$ is amplified by $60 \mathrm{~dB}$ with a two stage amplifier. The first stage gives a gain of $40 \mathrm{~dB}$ with a low noise amplifier and a gain of $20 \mathrm{~dB}$ in the next stage is prvided by general purpose op-amp. The signal is then fed to a comparator which compares the amplified echoed signal to a reference voltage. Any false reception should have amplitude less than the reference value and should not be considered to be a valid echo. The data processing and storage block incorporates a microcontroller for processing tasks and an external EEPROM for the storage. PIC $18 \mathrm{f} 4550$ is chosen due to its 1310 -bit A/D channels and the special feature of USBv2.0 compliance which makes it possible to use in HID (Human Interaction Device) mode when troubleshooting or reading the data stored in module. Even though this particular chip has 256 bytes built-in EEPROM, external mode of storage is chosen due to large amount of data to be accumulated during operation by node modules. As the velocity of sound is dependent on temperature, a temperature compensator circuit is employed which provides the conversion value that corrects the error generated by the change of sound velocity due to change in temperature. The conversion error becomes critical when the distance to be measured becomes long enough which sets a limit on the measurable distance by the module. A temperature sensor is employed to sense the temperature depending on which the calculation of water column height is optimized.

2) Network interface: To connect any module to the network and to make it able to send data over wireless network, RS232 interface is used. This section includes Microcontroller Unit (MCU), a GPRS enabled GSM modem and RS-232 level converter. This interface is controlled by the MCU. The control over communication module ensures conservation of power while in off-line mode. The modem is connected to the MCU to receive AT (Attention) commands from the MCU to connect to the GSM/GPRS network. The modem uses a SIM (Subscriber Identification Module) from a network service provider with GPRS enabled. The measured data is sent as packets using GPRS. Before sending, data are encapsulated in pre-defined format with a header that identifies a particular node in the network. This is a two digit decimal ID that separates among different sending nodes.

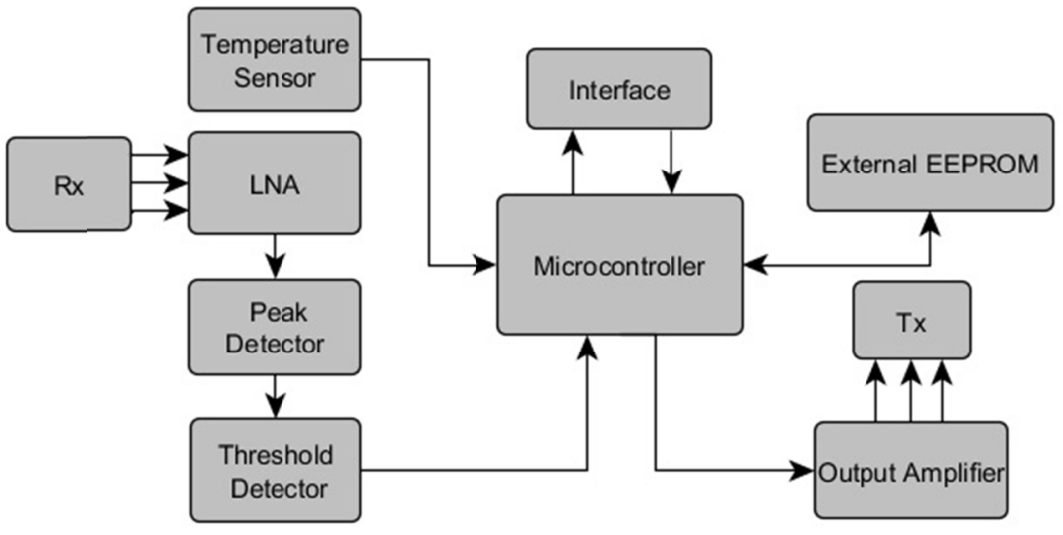

Figure 4. Architectural block diagram of system hardware 
3) Real Time Clock (RTC) generation: To stamp the events in real time, there is a real time generation section. This is actually a RTC integrated circuit (DS1307) employed to operate in $\mathrm{I}^{2} \mathrm{C}$ slave mode over a two-wire serial bus with MCU acting as the master. The design is done according to the application note provided by Maxim Integrated Products (2006). Timing information is stored in 56 bytes of NV (Non-Volatile) SRAM from where it is fetched by master when necessary.

Along with these sections, a few voltage divider networks are used to feed voltage signals to MCU. This section actually monitors different parameters from other sections, e.g. node battery voltage. The voltage is converted by MCU and sent to the server along with other data.

\subsection{Explanation of Acquisition Process}

When the system is switched on, MCU initializes the modules necessary for system operation. After the initializations are being done, GLOBAL interrupt is enabled to allow the external interrupts. The contents of count register of TIMER1 and capture register are cleared. MCU starts sending $40 \mathrm{KHz}$ pulse whose transmission length can be varied from the firmware. After a transmission pulse is sent, there is a chance that the transmitted signal could directly fall on the receiver. This could trigger a wrong detection. To avoid this scenario, the signal from the echo detector is disregarded until a certain period of time is elapsed which ensures the avoidance of reception of the transmitted pulse directly instead of the echoed signal. MCU waits $1 \mathrm{~ms}$ and then enables the detector circuit. Just prior to this, the capture module and timer modules are turned on. TIMER1 starts to count the number of pulses between the turn on and another pulse to indicate the end. When an echo is received, the capture module turns off the timer module and the count value is stored. From the timer count, the time taken by the echoed signal to traverse the length of the tube above water is calculated. If the time taken by the sound wave to return to the module is $t$ seconds, the one way distance traversed by the transmitted wave before being captured by $\mathrm{R}_{\mathrm{X}}$ will be: $l=(v \times t) / 2$, where $v=$ velocity of sound at a specific temperature. If the clock of TIMER 1 is configured as to count $1 \mu \mathrm{s} /$ count, the value of the TIMER 1 count register will indicate the number of instances elapsed since US wave is being transmitted. It takes $k$ microseconds to execute one instruction for a PIC MCU with an $F$ MHz clock where, $k=1 / F$. This count value is used as the value of $t$. Hence, the TIMER 1 module must be configured very carefully depending on the system clock. Before transmitting the signal, MCU samples the temperature sensor data to get the instantaneous temperature value and sets the value of $v$ to be used in that calculation. The dependency of velocity of sound on temperature is governed by this formula:

$$
c=331 \mathrm{~ms}^{-1}+0.6 \mathrm{~ms}^{-1} \mathrm{C}^{-1} \times T^{\circ} \mathrm{C}
$$

Where $T$ is temperature in degree Celsius. A lookup table (LUT) containing values of velocity for every $1{ }^{\circ} \mathrm{C}$ increase in temperature between the range $5{ }^{\circ} \mathrm{C}-50{ }^{\circ} \mathrm{C}$ is used by firmware after acquiring temperature revision data for appropriate value of $v$. The actual water column height is determined afterwards which is stored and sent to remote server. The whole system process is illustrated in Figure 5.

\subsection{Node firmware}

The software works in two successive steps: one is for acquiring column height and another for storing and sending the data over internet. MCU is loaded with software to carry on the signal generation, reception, data acquisition, data storage and data transmission tasks. The coding steps can be visualized with the help of a flow chart which is shown in the Figure 6. After the system is switched on, built-in timer module TIMER0 is initialized with a calculated pre-scalar so that the timer counts up to 65535 which indicates a timeout of $65 \mathrm{~ms}$ at $4 \mathrm{MHz}$ clock speed used for controlling purpose of transmission period. TIMER1 is initialized to capture the echoed signal from the slab surface. Capture module CCP1 is turned off to prevent the module from capturing noise. A temperature revision data is input to $\mathrm{A} / \mathrm{D}$ channel which is used to get the current velocity of sound from a LUT. As the river surface should have some disturbances in terms of current and waves, there must be situations when the successive readings will yield data which are different from each other significantly. To mitigate this effect, multiple reading are taken during a single measurement and then averaged to get a value that lies in between the maximum and minimum values with minimum aberration from the true value. A variable $r$ is declared to have a null value that indicates the number of readings taken. Enabling the interrupts, MCU declares another variable $k$ which is also assigned a null value at the beginning of a process. The limiting value of $k$ defines the number of readings per measurement cycle to be made. Signal transmission is initiated by MCU and continues for a period of $0.5 \mathrm{~ms}$. Reception unit is turned on $1 \mathrm{~ms}$ later to eliminate the wrong reception of the transmitted pulse instead of echoed one. Capture mode is initiated by enabling CCP1 module. Interruption can be caused by either TIMER 0 overflow or echo reception. If TIMER0 is overflowed, MCU restart the TIMER and reinitiate pulse sending sequence. A distance of $(65535 \times v) / 2$ should be covered by the transmitted pulse train 
before the timer is overflown, so a bias of $(65535 \times v) / 2$ is added to the final value of calculated distance. A valid interrupt from capturing the echo makes MCU to get the capture value and initiate the calculation to get the actual height of water column. The temperature data and capture value yields the vertical distance between the transmitter-receiver pair and the slab surface using the formula disused in section 2.1. The capture value is added to the time being waited before enabling pulse reception to account for the true time gap between the two events. After the calculation, $k$ is incremented by unity and if it holds a value less than 2000 , the whole process starting from the initiation of pulse transmission repeats. After 2000 readings, MCU calculates the final distance by averaging all the readings. The current time is fetched from RTC and data is time stamped by writing that time and data in successive memory locations in EEPROM.

Prior to going in to the transmission MACRO, another A/D conversion takes place to get the current status of the node power source in terms of percentage of power left. Then MCU goes into the transmission MACRO. Transmission MACRO prepares the modem for sending the collected information to the remote server and handles the protocol for doing so. The data set consists of 2 extra bytes which include one node id byte and one measurement sequence id byte. Once the send command is issued, each data byte is sent and a timer of one hour is set. MCU then waits for the ACK (Acknowledgment) from the server to arrive. If the ACK arrives before the timer expires, MCU disables the communication module and steps out of the MACRO. If timer runs out, the whole set of data is sent again and the communication module is disabled regardless of ACK is received or not. Sequence variable $r$ is then incremented by unity and MCU steps into a wait state which lasts till the beginning of next measurement cycle. Before commencing another measurement, $r$ is checked to have a value equal to or less than 12. This limiting value ensures the number of measurements within a period of 24 hours is to be 12 . A value greater than 12 makes MCU to go to the top of the execution list and to clear the variable. Sensor hardware operates in two basic modes: low priority and high priority modes. The modes are dependent on the rate of change between two successive measurements. If the rate of rise (RR) is positive and greater than a threshold value, the firmware switches to the high priority level and re-initializes the value of $r$ to be greater than 12 . This, in consequence ensures a more frequent sequence of measurements. The final wait state is adjusted according to the value of $r$.

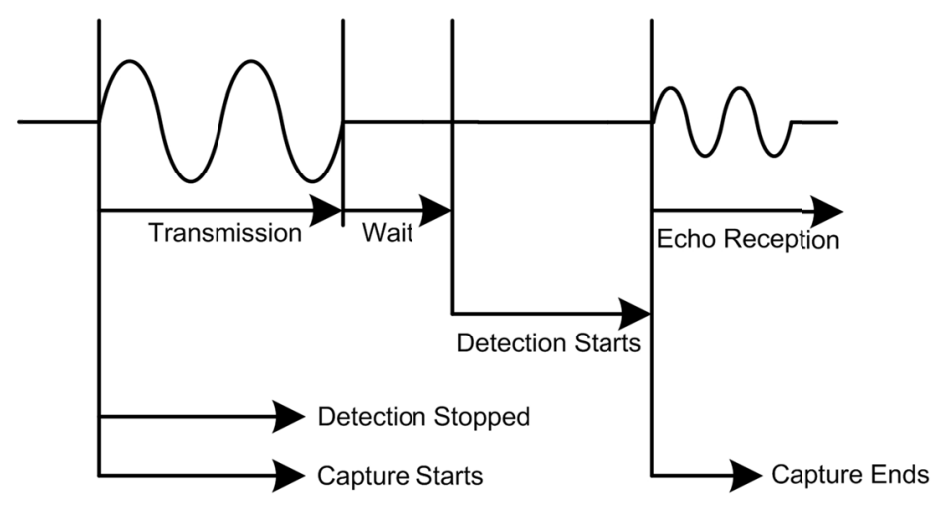

Figure 5. Process time line 


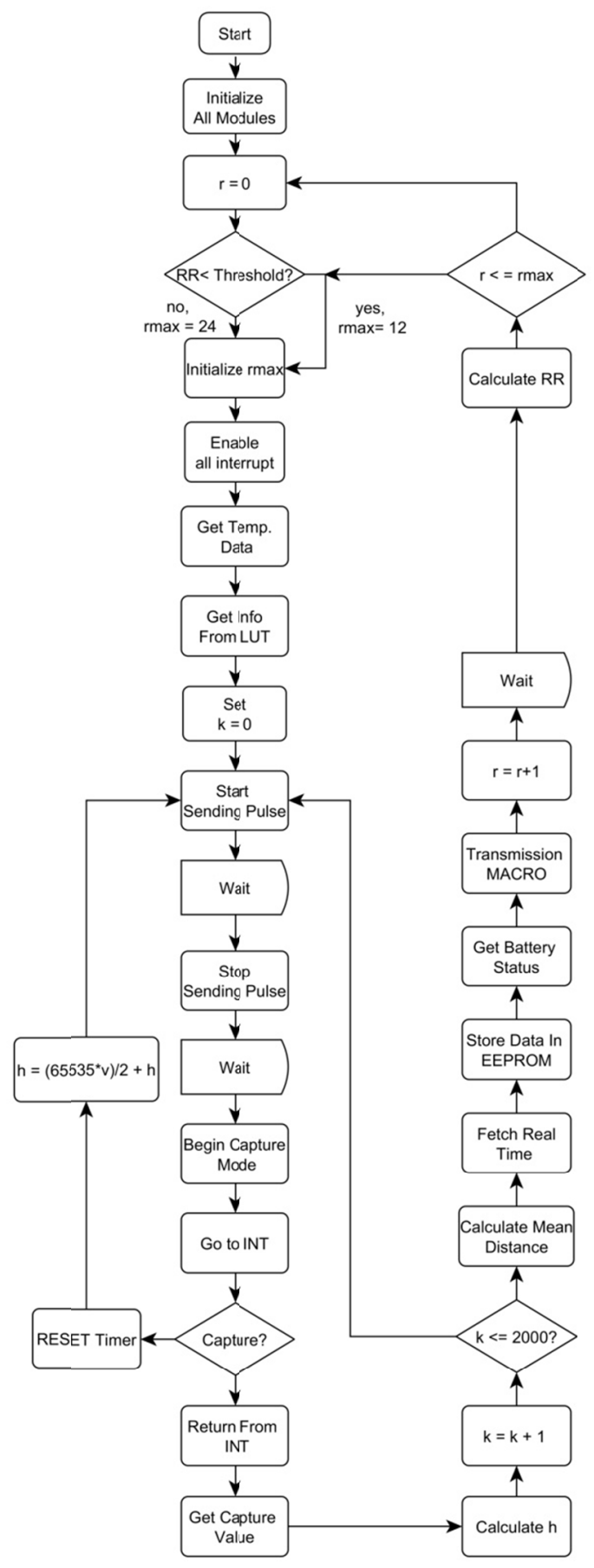

Figure 6. Node software flowchart

\section{Central Monitoring Unit (CMU) and Server Algorithms}

CMU consists of a hardware section along with an algorithm running with a database. The database GUI is written using Visual Studio. A graphical description of CMU is given in Figure 7. The receiver site algorithm follows the stop-n-wait ARQ (Automatic Repeat Request) protocol. This protocol is well suited for noisy channels and increases the reliability of the communication as suggested by Forouzzan (B. A. Forouzan, 2009). Server waits for a packet to arrive. When a packet arrives and the data is not corrupted, it extracts the information and delivers to the database. The database enters the data using the node id and measurement sequence. $R_{n}$ is the sequence number assigned for data arrived at the site. For each node, a separate $R_{n}$ sequence is maintained. This helps to detect any missing data when the received data do not contain the expected sequence number. This kind of scenario generates an error message to indicate any disturbance during transmission. Each successful extraction of information causes $R_{n}$ to increase by unity. Prior to sending an acknowledgement to the 
corresponding node, it checks to see if the sequence count exceeds twelve. The rate of rise is monitored and if it is greater than the threshold defined for a particular node, CMU expects the node to change the maximum count per day to be changed to a value greater than 12. The algorithm is illustrated in Figure 8 .

\subsection{Event Prediction Algorithm}

There are some useful models for developing a successful event prediction algorithm. The challenge is to choose the one that would produce a result that matches closely with the actual data. The model of prediction algorithm is chosen after careful evaluation of the following three different techniques (Chapara \& Canale, 2006; Ross, 2007).

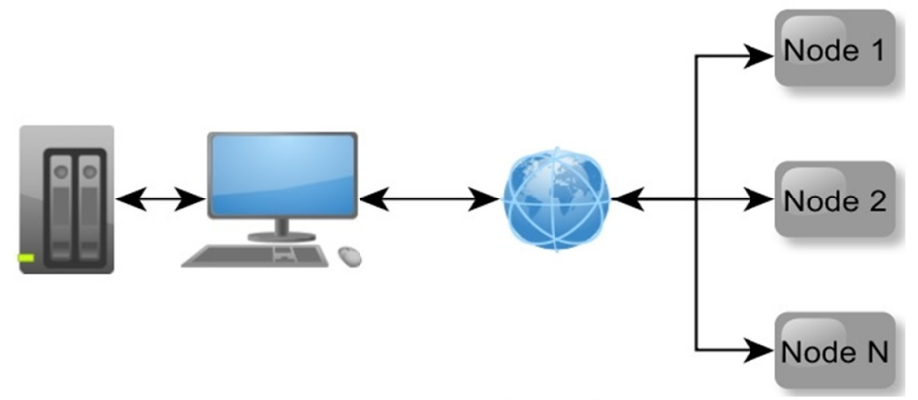

Figure 7. CMU general block diagram

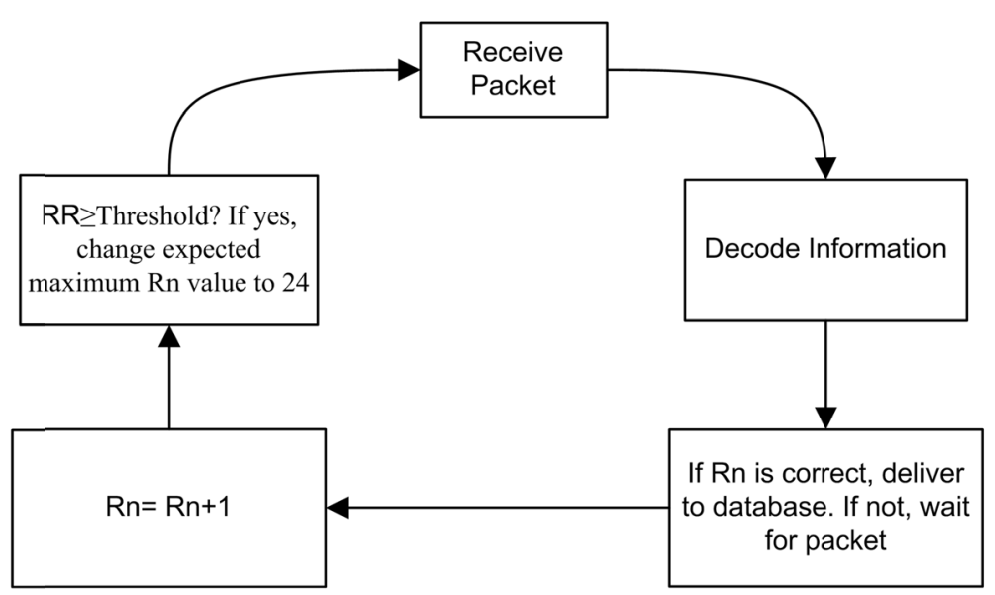

Figure 8. CMU process cycle

1) Linear regression: If there is a straight line containing a set of paired observations- $\left(x_{1}, y_{1}\right),\left(x_{2}, y_{2}\right) \ldots \ldots\left(x_{n}, y_{n}\right)$; the mathematical expression for the straight line is: $y=a_{0}+a_{1} x+e$; where $a_{0}$ and $a_{1}$ are coefficients and $e$ is the residual between the model and the observations. The sum of the squares of residuals between the measured $y$ and the $y$ calculated with the linear model is minimized to get the best fitted data set. This is a fairly good approach for prediction of future events but with a constraint; which is that the data set must maintain a linear relationship. The observations may or may not have a linear behavior; hence there is always a margin of error.

2) Second order regression: As the observations could exhibit marked patterns this would be poorly represented by straight line. For this kind of cases, a curve would be best suited to fit data. An approach would be to fit an appropriate polynomial to the data using polynomial regression. Using a quadratic equation to describe the water level states, a set of data is obtained and compared with the actual data.

3) Markov model: Hidden Markov model (HMM) is adopted as the third solution. HMM is a well-practiced model to predict faults and failures in systems. In our system, we used it to model a predictive behavior for generating a sequence of most likely events where all the observations are treated as a failure, according to the technique proposed by F. Salfner, P. Tröger, and S. Tschirpke (Salfner, Tröger, \& Tschirpke, 2009). A hidden Markov model is an extension of a finite discrete time Markov chain (DTMC). DTMCs are defined by a set of labeled states $\mathrm{S}=\{\mathrm{Si}\}(1 \leqslant \mathrm{i} \leqslant \mathrm{N})$, a square stochastic matrix $\mathrm{A}=\left[a_{i j}\right]$ defining transition probabilities between any pair of states, and a vector $\pi=\left[\pi_{i}\right]$ specifying an initial probability for each state. Hidden Markov models 
extend DTMCs in that an output is produced each time the DTMC enters a state. The occurrence of failures can be predicted by identifying special patterns of errors the system is experiencing.

Each of these techniques is used to implement three different models based on the average of observations of each day for 30 days. The adequacies of models were tested by plotting the predictions calculated by the model against the average of acquired data for 5 days. The comparative predictions are shown in Figure 9. From the bar diagram, it is evident that HMM has yielded prediction those experienced a minimum deviation from true values.

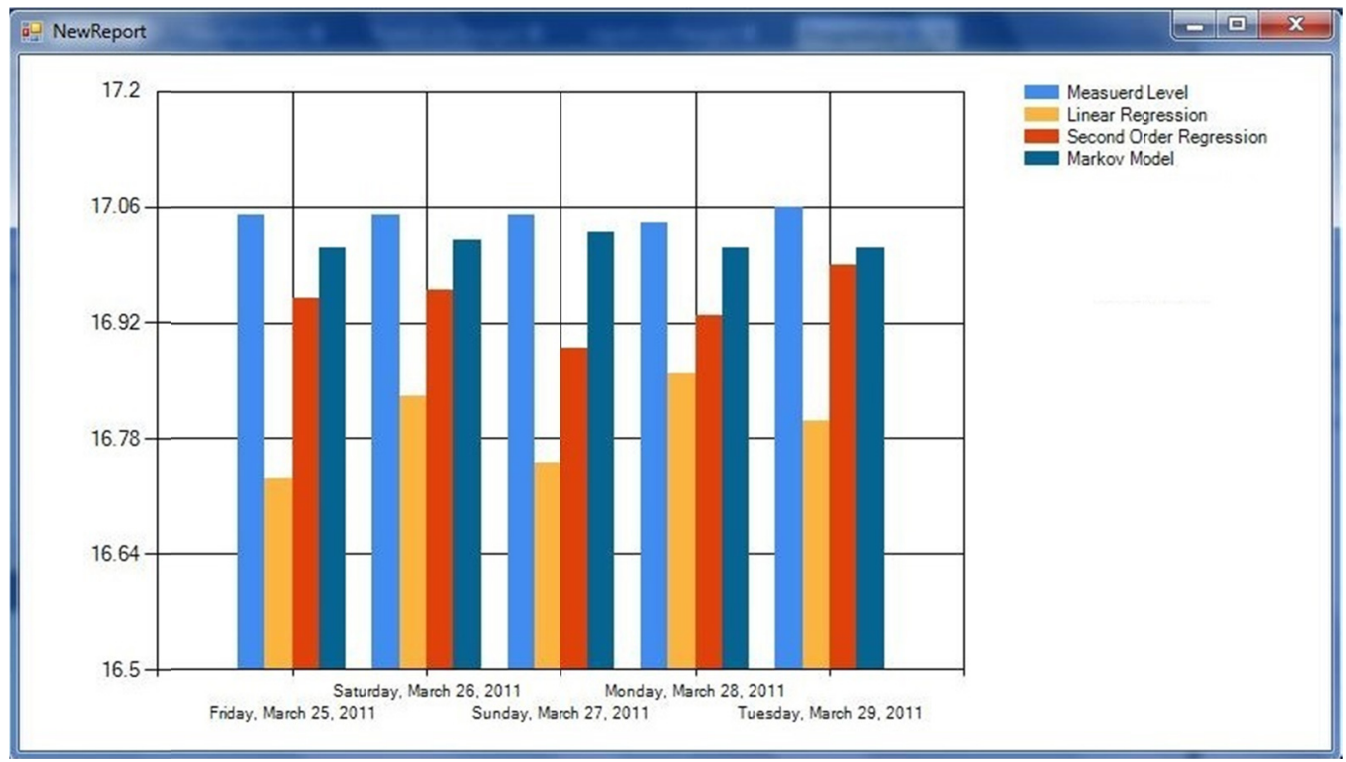

Figure 9. Bar diagram showing comparison among predictions using different models

Due to least amount of aberration from the actual data which are acquired manually, HMM is chosen for developing the prediction algorithm.

\section{Results \& Discussions}

\subsection{Prototype Development and Installation}

Prior to the installation of the modules at node sites, the distance measuring capability of prototype module is verified at the laboratory test environment for horizontal and vertical paths with a minimum of errors. As the measurable distance of data acquisition systems is between $0.3 \mathrm{~m}$ to $3.0 \mathrm{~m}$ in the prototype designed during previous work (Chowdhury et al., 2011), node modules cannot be mounted more than $3.0 \mathrm{~m}$ above the water surface. This limit imposed a drawback on the tube length used at various node points. The modification in the firmware to use the timer overflow mechanism to increase the measurable distance solved this particular problem. The density factor in determining the water level brought more precision in measurement than the previous system. The whole system is assembled and six nodes are placed at six different points of three different rivers: Buriganga, Dholessori and Turag, with two nodes along each river. All these rivers have an average depth of 25-35 meters and have recent records of causing flood. The months of March-August are chosen to be the test time window as this is the time of the year when the rivers are not too deep or too shallow. Another reason for choosing this window is that during this time the depth of rivers are subjected to continuous and rapid changes due to heavy rain. Near the bank of these rivers, depth is fairly less and GSM network is accessible by the nodes. For these reasons, places near the banks are qualified as the installation sites which provided sufficient depths. The installation sites were chosen not to be too crowded and noisy such as harbors as there might be noise that can interfere with the US signal and thereby degrade the performance. The feature of monitoring power sources at the node allows for the timely replacement of the power source in nodes. However, if any node is shut down due to power failure, it could be useful by reading all the data for that particular day from EEPROM so that no data is lost.

\subsection{Measurement Accuracy}

The principal concern with any measrement system is the accuracy of the measurement because of the fact that the performance of the system will be solely depenedent on the degree of closeness of the measured value to the actual value. To determine the accuracy yielded by the prototype system, initially we set up a single node, 
collected water level data manually and compared with the data sampled by our system. Both measurements were made concurrently so as to ensure the clarity of evaluation. A total of 100 samples were collected during 3 days time and compared to determine the degree of closeness. Figure 10 illustartes the distribution of percentage error over these 100 samples and the average error calculated.

\subsection{Measurement Results}

The screenshot of entry window of the database software in Figure 11 (a) shows all the nodes with their names,

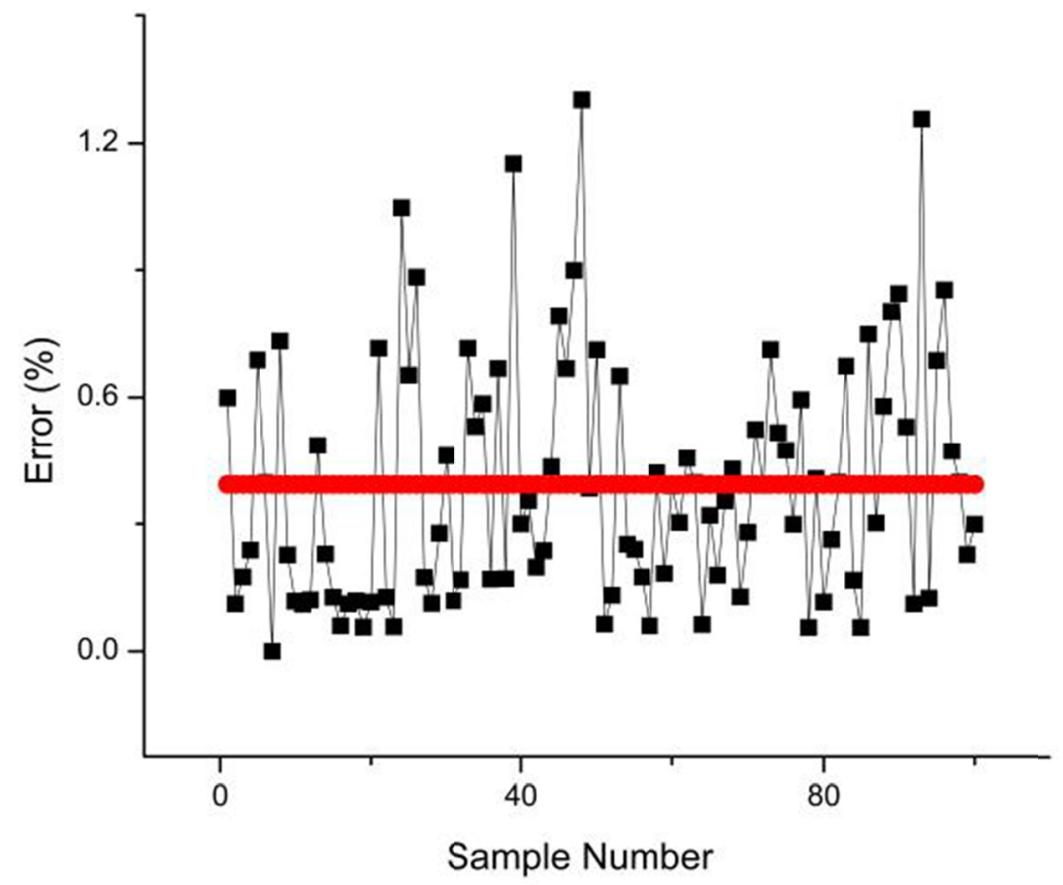

Figure 10. Illustration of accuracy of measurement by percentage error over 100 samples. The red line represents the average value of error experienced 


\begin{tabular}{|c|c|c|c|c|c|}
\hline server_time & node_time & measured_level & river & node_id & power_status \\
\hline $10: 00: 25$ & $10: 00: 12$ & 16.74 & Buriganga & 1 & $67 \%$ \\
\hline $10: 00: 53$ & $10: 00: 47$ & 15.08 & Turag & 1 & $65 \%$ \\
\hline $10: 01: 04$ & $10: 00: 58$ & 16.53 & Dholesshori & 1 & $67 \%$ \\
\hline $10: 01: 10$ & $10: 01: 03$ & 17.05 & Buriganga & 2 & $68 \%$ \\
\hline $10: 01: 21$ & $10: 01: 13$ & 18.54 & Dholesshori & 2 & $66 \%$ \\
\hline $10: 01: 29$ & $10: 01: 22$ & 15.94 & Turag & 2 & $65 \%$ \\
\hline $12: 00: 15$ & $12: 00: 11$ & 16.71 & Buriganga & 1 & $66 \%$ \\
\hline $12: 01: 03$ & $12: 00: 59$ & 16.56 & Dholesshori & 1 & $65 \%$ \\
\hline $12: 00: 52$ & $12: 00: 47$ & 15.11 & Turag & 1 & $64 \%$ \\
\hline $12: 01: 17$ & $12: 01: 12$ & 18.54 & Dholesshori & 2 & $65 \%$ \\
\hline $12: 01: 09$ & $12: 01: 03$ & 17.03 & Buriganga & 2 & $66 \%$ \\
\hline $12: 01: 26$ & $12: 01: 22$ & 15.96 & Turag & 2 & $63 \%$ \\
\hline $14: 03: 05$ & $14: 01: 00$ & 16.67 & Dholesshori & 1 & $64 \%$ \\
\hline $14: 00: 15$ & $14: 00: 11$ & 16.89 & Buriganga & 1 & $65 \%$ \\
\hline $14: 00: 52$ & $14: 00: 47$ & 15.26 & Turag & 1 & $63 \%$ \\
\hline $14: 01: 07$ & $14: 01: 03$ & 17.17 & Buriganga & 2 & $64 \%$ \\
\hline $14: 03: 21$ & $14: 01: 22$ & 16.04 & Turag & 2 & $62 \%$ \\
\hline $14: 01: 20$ & $14: 01: 12$ & 18.67 & Dholesshori & 2 & $64 \%$ \\
\hline $16: 00: 22$ & $16: 00: 12$ & 16.84 & Buriganga & 1 & $63 \%$ \\
\hline $16: 01: 07$ & $16: 01: 01$ & 16.65 & Dholesshori & 1 & $63 \%$ \\
\hline $16: 00: 55$ & $16: 00: 47$ & 15.23 & Turag & 1 & $63 \%$ \\
\hline $16: 01: 14$ & $16: 01: 07$ & 17.14 & Buriganga & 2 & $63 \%$ \\
\hline $16: 01: 34$ & $16: 01: 22$ & 16.01 & Turag & 2 & $60 \%$ \\
\hline $16: 01: 27$ & $16: 01: 11$ & 18.63 & Dholesshori & 2 & $63 \%$ \\
\hline
\end{tabular}

(a)

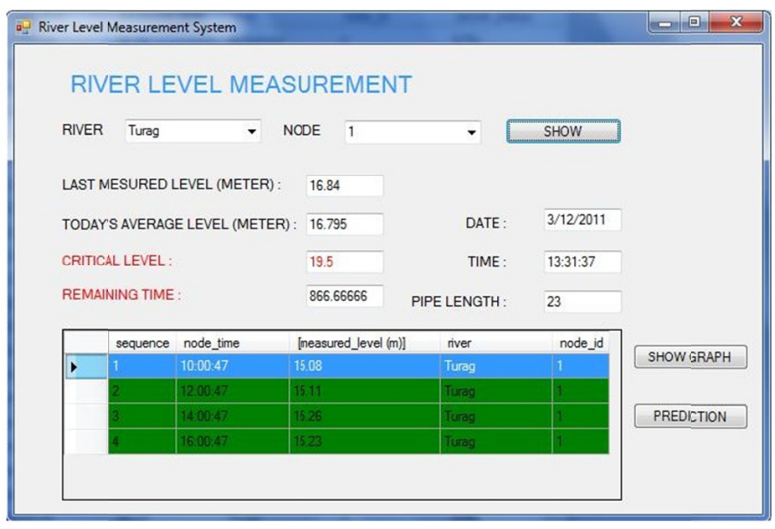

(b)

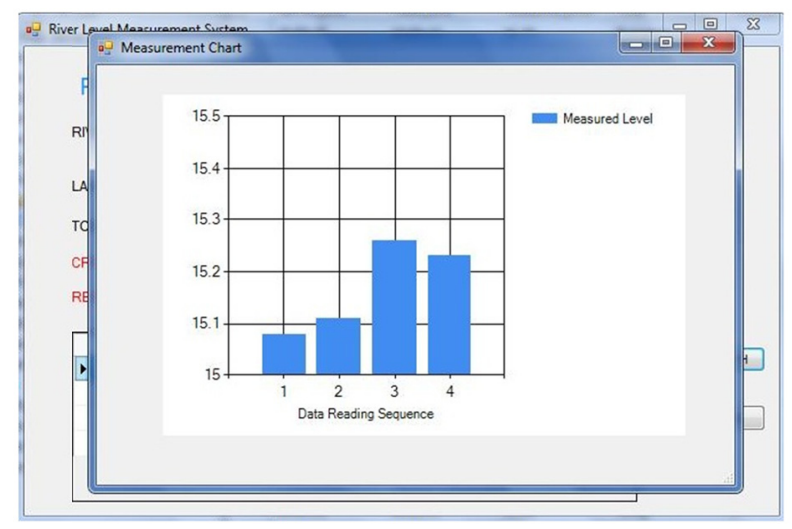

(c)

Figure 11. (a) GUI window showing database entries with node parameters (b) Node details window (c) Measurement graph window

last measured levels, server time of reception of data from the nodes, node time (real time) for data collection etc. in order of reading sequence. The node power status represents the percentage of power left on the node. More than ninety five percent of data are received within 20 seconds from the beginning of measurement cycle. The delayed data were sent by the nodes at busy hour which explains the delay. The window of Figure 11 (b) shows the details of a particular node with information associated with that node e.g. river name, node id, length of the pipe used and the measured levels of that node along with the node times etc. Each reading is marked with green background which turns red upon receiving a status of power level available in that particular node which is less than thirty percent. This window also contains the option for showing a graphical interpretation based on last twelve measurement received. The graphical representation of the measurements is shown in the window of Figure 11 (c). Figure 12 (a-d) illustrates the details from two more nodes, namely node 1 from Dholesshori river and node 2 from Buriganga river.

The real time monitoring produced better result than previous system as it gives the estimated time in real time instead of the time when the data are received by the server. Also, the multiple measurements during a single session and the use of arithmetic mean reduced the effects of disturbances on the water surface. The periodical 
ups and downs in the water levels were due to the periodic high tide and low tide which is natural. In twenty four hour window, maximum 12 and minimum 11 data were received from the nodes. We analyzed that the loss of measurement data were during peak hour (i.e. $11 \mathrm{am}-2 \mathrm{pm}$ ) which explains the data reception success rate of $95.83 \%$. However, this is the worst case scenario as this represents the minimum success rate. When a 15 days window is considered, the success rate rises up to $98.89 \%$ with only 2 additional measurement data loss during that period. The lost measurement data were later retrieved from the node and used in statistical analysis of the sampled data. The critical time (time to exceed the critical level) is updated once per day. The time to exceed the critical level is calculated based on the average of the all the sample data received on any day.

Figure 13 shows the event prediction bar diagram for an individual node. The algorithm running at the server offers the user with the average levels for the next five days. The measured values from the nodes are compared against the manually measured values to determine the measurement acuracy. We have used modified standard deviation to determine the statistical accuracy of the measurement samples found from the nodes. In our analysis, we used the average of actual measurement from that particular node as the average value from which the node samples are deviated. As each day is susceptible to different standard deviation values of sample from any node, an arithmetic average of these standard deviations is used to determine the standard deviation for that particular node during a 15 days period. The changes in everyday standard deviation is depicted in Figure 14 which illustartes that the performance of the devised network in terms of measurement accuracy and as well as precision graphically. The average standard deviation is below 0.6 which suggests very good accuracy of the acquired data. Also the red line representing the actual standard deviation calculated from measurements at each day oscillates between 0.2 and 0.7 which indicates that the precision of measurement of data is satisfactory.

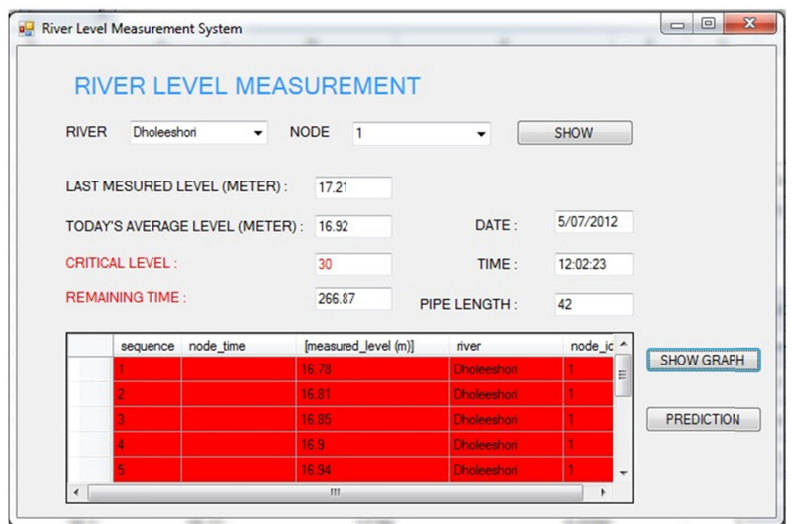

(a)

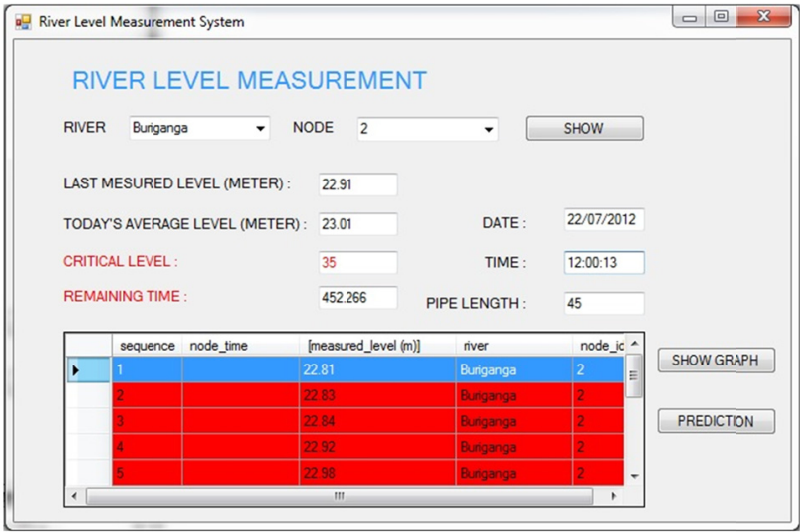

(c)

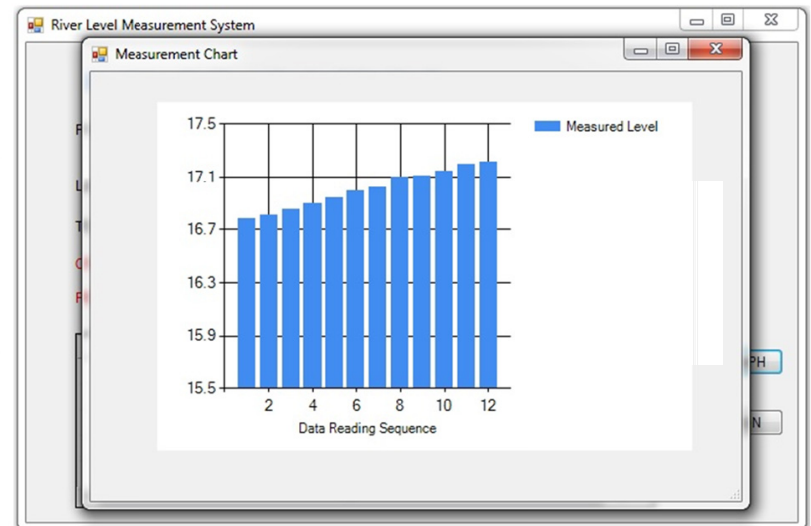

(b)

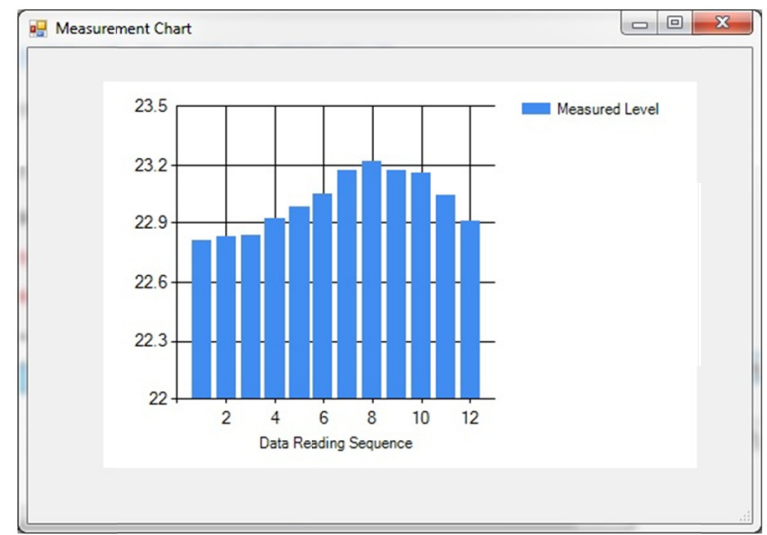

(d)

Figure 12. Database entries (a and c) from two more nodes with respective GUI windows showing database entries (b and d)

\subsection{Probable Drawbacks of Prototype System and Solutions}

During the deployment process of this prototype system, the installation sites were chosen to be at the close 
vicinity of the banks of the rivers so that GSM network can be utilized for sensor networking. This is a challenging task featuring wide geographic network coverage, highly dynamic radio transmissions, high end to end packet delivery rate requirements and hostile system deployment environments. The method of connectivity to the network is of utmost importance here. One solution would be to arrange the nodes in a distributed mesh network where each node will be connected to a land based connecting node by another connecting wireless network other than GSM. The land based node would serve the purpose of transferring the acquired information from each node to $\mathrm{CMU}$.

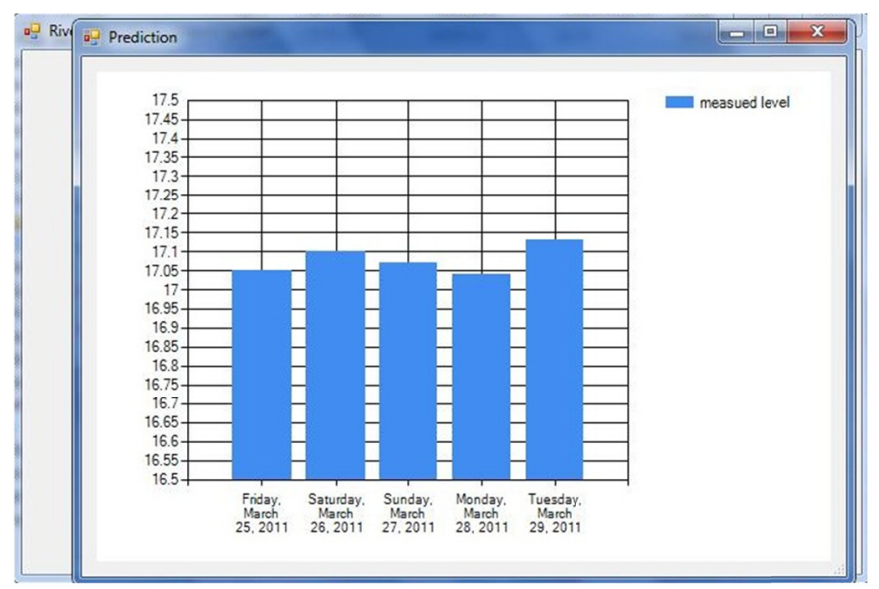

Figure 13. Prediction graph window

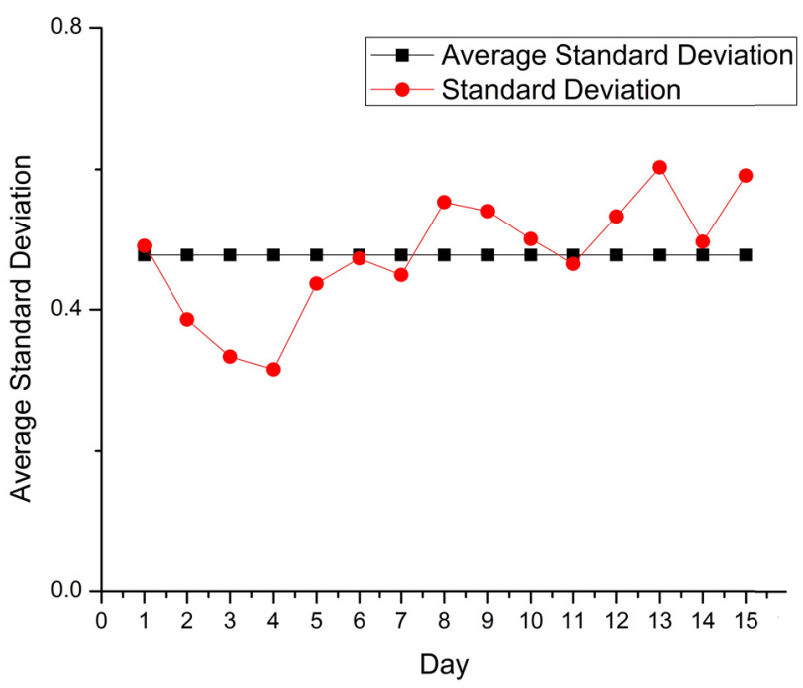

Figure 14. Standard Deviation of measurements in 15 days window

Also, the dependence of velocity of sound on only temperature is considered. Actually the parameter is also dependent on atmospheric pressure and humidity. In rough conditions of weather, these two parameters could change drastically over a short period of time and hence affect the velocity of sound. This, in effect could result in less accurate measurements. Sensing air pressure and relative humidity along with ambient temperature would be useful in determination of true value of height of water column.

\section{Conclusion}

The results show that the sensor network can provide predictability about the possible flood occurrence and helps to estimate a timeframe for taking necessary measures to mitigate damages of human life and properties. For further research, places where GSM network is not existent can be chosen. For those cases, advanced wireless communication protocols standardized by IEEE such as ZIGBEE (IEEE 802.15.4) could be used in signal hoping network architecture. For DAMs to measure distances more accurately in a noisy environment such as ports, marine harbor etc. digital polarity correlator suggested by a group of scientists (Nakahira, Kodama, 
Morita, \& Okuma, 2001) could be proved to be a solution. USB modem can be a great replacement in this system for utilizing $3 \mathrm{G}$ (Third Generation) connectivity for faster and more reliable networking. Firmware for USB connected modem could also reduce the power and spatial concerns for RS232 supported modems. The data analyzed by the server can find additional uses in other applications and researches also. The nature of water level of any particular river in each season can be taken into account to plan for the agricultural aspects of the nearby localities. Architects can decide whether the place under consideration is reachable to flood and design structures accordingly. Geographically important information such as time of high tide, low tide and any behavior other than normal could be extracted from the analyzed data. Using multi sensor-multi node technique in single installation site, change in depth of river bed can be understood. Advanced statistical analysis of the information could provide pattern of river level rise and fall over a long period of time which could be useful in understanding the effects on sea level by phenomenon like global warming. With sufficient data, a database could be built to observe the current scenario of the water level rise over a period of time which could give us a fair idea about the effect of global warming over a particular geographical area.

\section{References}

Cardell-Oliver, R., Kranz, M., Smettem, K., \& Mayer, K. (2005). A reactive soil moisture sensor network: Design and field evaluation. International Journal of Distributed Sensor Networks, 1(2), 149-162. http://dx.doi.org/10.1080/15501320590966422

Chapara, S. C., \& Canale, R. P. (2006). Numerical Methods for Engineers (5th ed.). New York, USA, McGraw-Hill.

Chowdhury, Z. I., Imtiaz, M. H., Azam, M. M., Sumi, M. R. A., Rahman, M. R., Alam, F., .. Hassan, N. (2011). Design and deployment of a robust remote river level sensor network (pp. 244-249), Proceeding of IEEE Sensors Applications Symposium. Texas, USA. http://dx.doi.org/10.1109/SAS.2011.5739776

Forouzan, B. A. (2009). Data Communication and Networking (4th ed.) New Delhi, India, McGraw-Hill.

Gueuning, F. E., Varlan, M., Eugene, C., \& Dupuis, P. (1997). Accurate distance measurement by an autonomous ultrasonic system combining time-of-flight and phase-shift methods. IEEE Transactions on Instrumentation and Measurement, 46(6), 1236-1240. http://dx.doi.org/10.1109/IMTC.1996.507414

Jonkman, S. N. (2005). Global perspectives of loss of human life caused by floods. Natural Hazards, 34, 151-175. http://dx.doi.org/10.1007/s11069-004-8891-3

Maxim Integrated Products. (2006). Using a DS1307 with a PIC Microcontroller. Retrieved from http://www.maxim-ic.com/app-notes/index.mvp/id/3921

Nakahira, K., Kodama, T., Morita, S., \& Okuma, S. (2001). Distance measurement by an ultrasonic system based on a digital polarity correlator. IEEE Transactions on Instrumentation and Measurement, 50(6), 1748-1752. http://dx.doi.org/10.1109/19.982975

Park, K. T., \& Toda, M. (1996). Short distance ultrasonic distance meter. U.S. Patent No. 5, 483, 501. Retrieved from http://www.google.com/patents/US5483501

Postolache, O. A., Girao, P. M. B. S., Pereira, J. D., \& Ramos, H. M. G. (2005). Self-organizing maps application in a remote water quality monitoring system. IEEE transactions on instrumentation and measurement, 54(1), 322-329. http://dx.doi.org/10.1109/TIM.2004.834583

Ross, S. M. (2007). Introduction to Probability Models (10th ed.), San Diego, USA, Academic Press.

Salfner, F., Troger, P., \& Tschirpke, S. (2009). Cross-core event monitoring for processor failure prediction. In High Performance Computing \& Simulation, 2009. HPCS'09. International Conference on (pp. 67-73). IEEE. http://dx.doi.org/10.1109/HPCSIM.2009.5191988

Winter, T. C., LaBaugh, J. W., \& Rosenberry, D. O. (1988). The design and use of a hydraulic potentiomanometer for direct measurement of differences in hydraulic head between groundwater and surface water. Limnology and Oceanography, 33(5), 1209-1214.

\section{Copyrights}

Copyright for this article is retained by the author(s), with first publication rights granted to the journal.

This is an open-access article distributed under the terms and conditions of the Creative Commons Attribution license (http://creativecommons.org/licenses/by/3.0/). 